differentiation and professional recognition of the condition more than 20 years ago.

Whatever your branch of adult or adolescent psychiatry, a significant percentage of your out- and in-patients will suffer from an underlying BPD. You will find ample information in this book to aid you in recognising the coexistence of a BPD in Axis I patients as well as those presenting with such problems as somatic complaints, eating disorders, depression, overdoses, substance misuse, trauma and self-mutilating behaviour.

The classification can be misused and this is well discussed. (Misuse can include under- and overdiagnosis.) Making an accurate diagnosis is really important because these patients suffer over a very long time, as do those professionals and family members who encounter them. Most importantly, there is increasing evidence (which is well-marshalled in this book) that, when an appropriate range of therapies are well coordinated and structured by specialist teams, a considerable reduction in disturbance can be achieved. Longerterm treatment with appropriately trained professionals leads to an increasing capacity to contain impulses, feelings and affects, with consequent improvement in social functioning. Without coordinated and skilled therapies the likelihood is that there will be a continuation of costly destructive and self-destructive behaviour and impoverished lives. Although the author is American, he is clearly well acquainted with important recent British contributions to understanding and treating BPD and groundbreaking research into the clinical effectiveness of such treatments.

This is a very readable book. It is scholarly, up to date and full of clinical wisdom (and vignettes). It is written from a broad empirical basis and therefore the place of medication, partial hospitalisation, family, group, cognitive and psychodynamic approaches are well covered and integrated. I would therefore recommend it to clinicians of all disciplines. A particularly useful section lists other relevant books, films, videos, newsletters and websites.

Familiarity with the book could be of considerable assistance in developing clinical governance recommendations and for audit projects and personal development plans. I hope that it might come to the attention of commissioning agencies who may be able to play an important part in guaranteeing that adult mental health services are appropriately organised to provide adequate services and skills to meet the treatment needs of patients with BPD.

Brian V. Martindale Consultant Psychiatrist in Psychotherapy, John Conolly Wing, West London Mental HealthTrust, Uxbridge Road, Southall UBI 3EU, UK

\section{The Ageing Brain}

By Lawrence Whalley. London: Weidenfeld \& Nicolson. 2001.182 pp. $€ 16.99$ (hb).

ISBN 0297645870

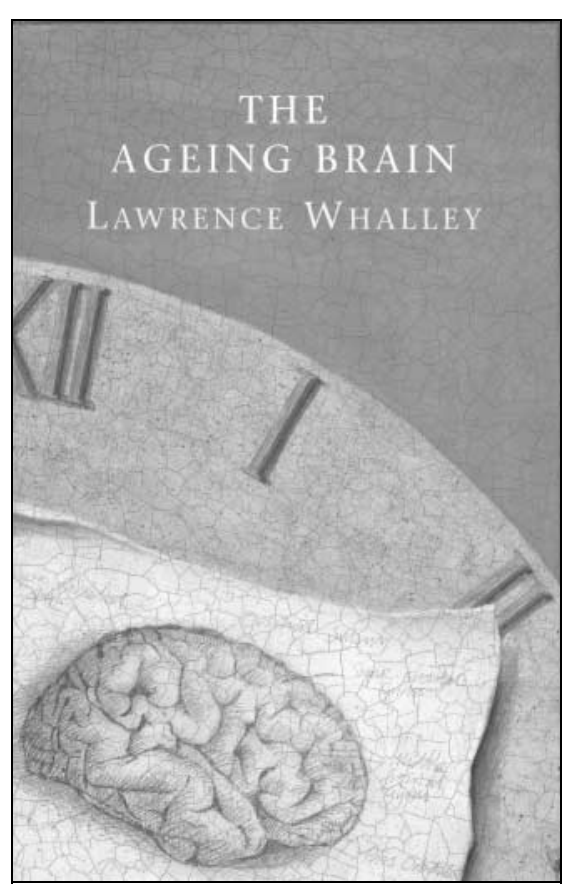

This book is part of the 'Maps of the Mind' series on brain research and would probably be classified as 'popular science'. Although the general reader would have little difficulty following the clearly explained concepts, the book is essential reading for anybody working in the field of old age psychiatry or neurology. The author is professor and head of mental health at the University of Aberdeen and states in his preface that one of his intentions is 'to counter the widespread pessimism about what the future has in store for us'. Beginning with a lucid discussion of the slippery concept of ageing, Whalley details physical, social and psychological aspects of growing older, and describes current thinking on the changes in mental ability which appear characteristic of normal ageing. After setting this solid and vivid scene of normality, he moves on to the dementias and their causes, chiefly
Alzheimer's disease and vascular pathology, and concludes with a plausible account of possible future developments in the treatment and perhaps prevention of these devastating disorders.

The book gives an excellent and readable account of brain function and dysfunction. Whalley manages to be concise and comprehensive at the same time, presenting potentially daunting hard science in an elegant style and including case studies to bring his concepts to life. His use of metaphor is impressive; for example, the mind is likened to a theatre, with various elements - audience, narrator, executive controller - representing different aspects of cognitive function. The scope is broad, encompassing cell biology, immunology, genetics, psychology and even virtual reality, and all in 182 pages.

Tim Stevens Lecturer in Psychiatry of the Elderly, Department of Psychiatry and Behavioural Sciences, University College London, London WIN 8AA, UK

\section{Memory Disorders in Psychiatric Practice}

Edited by German E. Berrios \& John R. Hodges. Cambridge: Cambridge University Press. 2000.520 pp. $€ 39.95$ (pb). ISBN 0 52I 576717

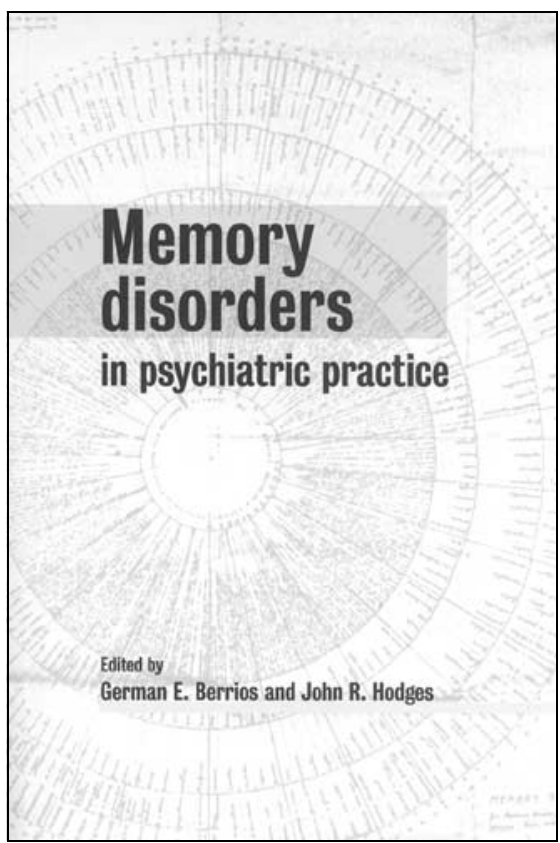

I reviewed this book with four criteria in mind: first, ease of readability; second, whether the knowledge base is appropriate 
for the target audience; third, whether any new knowledge is added to what is already known about memory disorders; and fourth, whether there is fair representation of the variety of opinions on memory disorders in psychiatric practice.

As regards readability, the labour of trudging through an over-inclusive first chapter almost discouraged me from proceeding to the much more interesting chapters that follow on mood and memory, the concept of meta-memory, and an interesting account of the psychopharmacology of memory.

In part two of the book, the editors and authors concentrate on the clinical and practical management of memory and its disorders, and this section is excellent. The description of the Cambridge multidisciplinary memory clinic gives insight into how an ideal memory clinic should function. However, the authors assume that other clinics will have similar levels of resources, which may not be the case. In many district general hospitals the approach may need to be tweaked to some degree.

Part three focuses on unique memory symptoms, with chapters covering paramnesias and delusions of memory, déjà $v u$ and jamais $v u$, confabulation, flashbulb and flashback memory. These rare but interesting syndromes are described in great detail. The chapter on functional memory complaints identifies two new syndromes - 'mnestic hypochondria' and 'functional cognitive disorganisation syndrome' - and makes the case for their inclusion in future classification systems.

This is an informative book. It will be a valuable practical reference for clinicians, psychiatrists, neurologists and clinical psychologists at a senior level in their training. It suffers considerably from being multiauthored. The style of writing varies from being over-inclusive to providing practical point-by-point summaries. However, it does cover a considerable proportion of what is known about memory disorders, and should be a valuable part of the library of memory disorders clinics.

Irshaad Ebrahim Specialist Registrar in Psychiatry, Neuropsychiatry and Memory Disorders Clinic, St Thomas' Hospital, Lambeth Palace Road, London SEI 7EH, UK 